Stellar Magnetism

C. Neiner and J.-P. Zahn (eds)

EAS Publications Series, Vol. ?, 2018

\title{
THE ROLE OF MAGNETIC FIELDS IN GOVERNING THE ANGULAR MOMENTUM EVOLUTION OF SOLAR-TYPE STARS
}

\author{
J. Bouvier ${ }^{1}$
}

\begin{abstract}
I review the development of ideas regarding the angular momentum evolution of solar-type stars, from the early 60 's to the most recent years. Magnetic fields are the central agent that dictates the rotational evolution of solar-type stars, both during the pre-main sequence, through star-disk magnetic coupling, and during the main sequence, through magnetized winds. Key theoretical developments as well as important observational results are summarized in this review.
\end{abstract}

\section{Introduction}

Magnetic fields hold clues to a number of properties of solar-type stars. Among those, their rotation rate is found to vary along the star's life, in response to a variety of physical processes, most of which are intimately related to the stellar magnetic field. In this review, I summarize what is currently known of the rotational evolution of solar-type stars and emphasize the central role magnetic fields play in governing angular momentum evolution. In Section 2, I briefly review the main steps that have led to the current understanding of rotational braking by magnetized winds on the main sequence. In Section 3, I describe more recent advances related to the angular momentum evolution of pre-main sequence stars and its relationship to magnetic star-disk coupling in young stars. In Section 4, these physical processes are illustrated through the development of angular momentum evolution models that grasp the main trends of the observed rotational evolution of solar-type stars, from their birth up to the age of the Sun. I conclude by mentionning a few theoretical and observational tracks that should provide an in-depth understanding of these issues in the years to come.

\footnotetext{
${ }^{1}$ Laboratoire d'Astrophysique, Observatoire de Grenoble, Université J. Fourier, CNRS, BP 53, 38041 Grenoble Cedex 9
} 


\section{Magnetic braking on the main sequence}

In an illuminating review paper, Kraft (1970) summarized what was known of the rotation rate of main sequence stars in the late 60 's. One of the main results was a clear break in the measured rotational velocities around a spectral type $\mathrm{F} 5\left(\mathrm{M} \sim 1.3 \mathrm{M}_{\odot}\right)$, with a striking contrast between rapidly rotating massive stars $\left(v_{r o t} \cdot \sin i \sim 40-150 \mathrm{~km} \mathrm{~s}^{-1}\right)$ and slowly rotating solar-type stars $\left(v_{r o t} \cdot \sin i \leq 25 \mathrm{~km} \mathrm{~s}^{-1}\right)$. Schatzman $(1959,1962)$ had anticipated this result by proposing a very efficient mechanism to remove angular momentum from latetype, magnetically-active stars. He suggested that the material ejected from the surface of solar-type stars, mostly through magnetic flares, could carry away a large amount of angular momentum, as the outflowing material is frozen in the stellar magnetic field and thus kept in near-corotation with the star up to large distances from the surface. The Weber \& Davis (1967) solar-wind model indeed predicts an angular momentum loss rate that scales with the square of the Alfvén radius, $R_{A}$,

$$
\frac{d J}{d t} \propto \frac{d M}{d t} \cdot \Omega_{\star} \cdot R_{A}^{2}
$$

where $\frac{d M}{d t}$ is the mass loss rate and $\Omega_{\star}$ the star's angular velocity, instead of scaling merely as the square of the stellar radius, $R_{\star}$, in the non-magnetized case. Since $\frac{R_{A}}{R_{\star}} \simeq 30$ for the Sun, the braking timescale by a magnetized wind is shorter by a factor of $\sim 10^{3}$ than what it is in the non-magnetized case. Magnetized stellar winds are thus quite efficient in braking late-type, magnetically-active stars on a timescale of $\sim 10^{8}$ yr during their early evolution on the main sequence (Belcher \& MacGregor 1976).

Another major result outlined in Kraft's (1970) review, was the fact that younger main sequence stars, as observed in young open clusters at an age of $\sim 100 \mathrm{Myr}$, tend to rotate faster than mature field dwarfs at an age of a few Gyr. Main sequence braking was further quantified by Skumanich (1972), who showed from the rotation rates of solar-type stars in the Pleiades, in the Hyades, and for the Sun, that the rotational velocity decreases on the main sequence as the inverse square-root of time, i.e., $v_{r o t}(t) \propto t^{-1 / 2}$. The so-called "Skumanich law" was readily interpreted in the framework of Schatzman's theory. With an angular momentum loss rate given by Eq. 2.1, and assuming the stellar magnetic flux is produced by a linear dynamo, i.e., $R_{\star}^{2} B_{\star} \propto \Omega$, one derives $\frac{d J}{d t} \propto \Omega^{3}$ (Durney \& Latour 1978), which integrates into $\Omega(t) \propto t^{-1 / 2}$. A general, albeit parametric, expression for the angular momentum loss rate by a magnetized wind has since then been worked out by Kawaler (1988).

As measurements of rotational velocities for solar-type stars on the main sequence became more abundant, it became apparent that the Skumanich relationship holds only on an average sense (Soderblom 1983). On the zero-age main sequence (ZAMS), at an age of about $100 \mathrm{Myr}$, solar-type stars actually exhibit a large intrinsic dispersion of rotational velocities, from less than $20 \mathrm{~km} \mathrm{~s}^{-1}$ up

to $150 \mathrm{~km} \mathrm{~s}^{-1}$ (Stauffer et al. 1985). This result was surprising indeed, as the extrapolation of the Skumanich relationship back in time from the age of the Sun 
to the ZAMS would predict velocities of order of $20 \mathrm{~km} \mathrm{~s}^{-1}$ at $100 \mathrm{Myr}$. How this wide distribution of ZAMS rotation rates builds up during pre-main sequence evolution will be discussed in the next section.

Angular momentum evolution models were developed, using the observed dispersion of rotational velocities on the ZAMS as initial conditions. The braking rate was assumed to scale with velocity as $\frac{d J}{d t} \propto \Omega^{3}$, so that fast rotators on the ZAMS are more efficiently spun down than slow ones during early MS evolution. As a result, the large dispersion in initial velocities is quickly reduced and converges towards uniformly slow rotation by the age of the Sun, in qualitative agreement with observations. It soon became clear, however, that a Skumanich type braking rate could not correctly reproduce the evolution of the rotational distributions observed for stars in open clusters of increasing age (cf. Fig. 1a). From empirical modeling, MacGregor \& Brenner (1991) suggested instead that such a braking rate applies to slow rotators only, and has a much shallower dependence on rotation for rapid rotators, above some threshold velocity, $\Omega_{\text {sat }}$.

Models using this alternative braking law were relatively successful in reproducing the observations (cf. Fig. 1b). In these models, the braking rate still scales as $\frac{d J}{d t} \propto \Omega^{3}$ for slow rotators $\left(\Omega \leq \Omega_{\text {sat }}\right)$, but only as $\frac{d J}{d t} \propto \Omega$ for rapid ones $\left(\Omega>\Omega_{\text {sat }}\right)$. This is equivalent to assuming a linear dynamo for slow rotators $(B \propto \Omega)$ that saturates at high rotation $\left(B \sim B_{\text {sat }}\right)$. While the actual physical meaning of "dynamo saturation" remains somewhat unclear, it is nevertheless supported by observations of chromospheric and coronal activity diagnostics, whose strength saturates at velocities larger than $10-20 \mathrm{~km} \mathrm{~s}^{-1}$ in young solar-type stars (Vilhu 1984; Stauffer et al. 1994). Furthermore, modeling the rotational evolution of lower mass stars suggests that the saturation velocity, $\Omega_{\text {sat }}$, depends on stellar mass, in such a way that saturation occurs at a fixed Rossby number, $R_{o}=\left(\Omega \tau_{\text {conv }}\right)^{-1}$, where $\tau_{\text {conv }}$ is the turnover convective time, over the mass range 0.5-1.0 $\mathrm{M}_{\odot}$ (Collier Cameron \& Jianke 1994; Krishnamurthi et al. 1997; Bouvier et al. 1997). This empirical result tends to provide some theoretical support to the concept of dynamo saturation in low-mass, partly convective stars.

Finally, the response of surface velocity to angular momentum loss at the stellar surface also depends on how efficiently angular momentum is transported in the stellar interior. A rigidly-rotating star has a longer spindown timescale than a differentially-rotating one in which only the outer convective envelope is braked while the radiative core remains in rapid rotation. This is because the moment of inertia of the convective envelope is much smaller than that of the whole star. Coreenvelope decoupling (Endal \& Sofia 1981) thus enters as an additional parameter in angular momentum evolution models. A physical description of angular momentum transport in stellar interiors was developed by the Yale group, with some success (Pinsonneault et al. 1989). Their rotational models, however, predicted a rapidly-rotating core for the present-day Sun, in conflict with heliosismological results. A more empirical approach was designed by MacGregor \& Brenner (1991), who assumed that both the radiative core and the convective envelope rotate uniformly, but not necessarily at the same rate $\left(\Omega_{\text {core }} \neq \Omega_{\text {conv }}\right)$. They introduced a coupling timescale between the core and the envelope, $\tau_{c}$, which measures the 

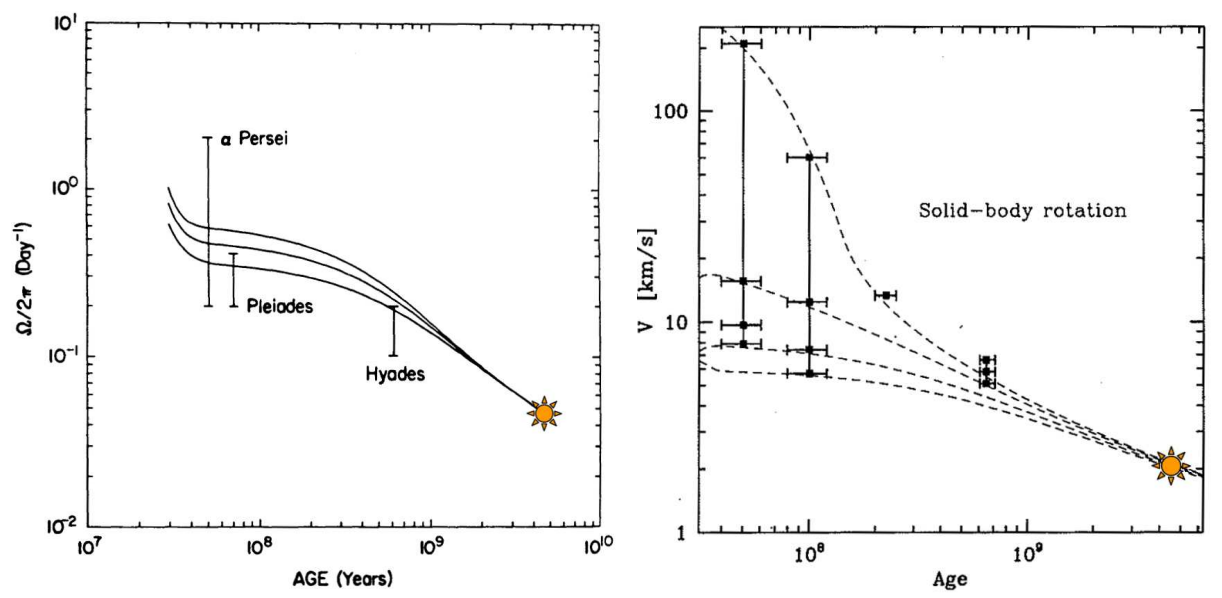

Fig. 1. Left : Angular momentum evolution models from the ZAMS to the age of the Sun, assuming a braking rate $\frac{d J}{d t} \propto \Omega^{3}$. The range of velocities observed for solar-type stars in 3 young open clusters is shown as bars. Models are shown for 3 initial velocities on the ZAMS. Adapted from MacGregor \& Brenner (1991). Right : Same as left panel but for models assuming dynamo saturation at $\Omega_{\text {sat }} \sim 14 \Omega_{\odot}$ (see text). Models are shown for 4 initial velocities on the ZAMS. The rotational distributions of solar-type stars in young open clusters are shown by vertical bars with 10, 20, 50 and 90 percentiles indicated. The remaining discrepancies between models and observations, most notably for slow rotators, arise from the assumption of solid-body rotation (see text). Adapted from Bouvier (1997).

time it would take to transfer angular momentum from the core to the envelope in order to restore uniform rotation throughout the star. A short coupling timescale enforces rigid-body rotation between the core and the envelope, while a long one leads to the development of a large velocity shear at the core-envelope boundary. Recent models based on updated observational constraints suggest that the coupling timescale itself depends on rotation, being much longer in slow rotators ( $100 \mathrm{Myr}$ ) than in fast ones ( 10 Myr) (Bouvier 2008, see Sect. 4).

Current models of angular momentum evolution for solar-type (and lower mass) stars on the main sequence thus rely on : i) initial conditions derived from measurements of surface rotation for ZAMS stars in young open clusters, ii) a parametrized braking law that includes dynamo saturation at high velocity, and iii) core-envelope decoupling. Unfortunately, no complete quantitative theory exists yet for angular momentum loss due to magnetized stellar winds, nor for angular momentum transport in stellar interiors. As a result, the models include these physical processes through simplified, parametrized relationships, with parameters being the normalisation of the magnetic braking rate (usually scaled on the Sun's), the velocity at which dynamo saturation occurs (somewhat constrained by the observations of magnetic activity diagnostics), and the timescale for angular momentum exchange 
between the core and the envelope (only loosely constrained by the Sun's internal rotation profile). The impact the various parameters have onto rotational evolutionary tracks is illustrated in Keppens et al. (1995), Bouvier et al. (1997) and Allain (1998). Combining these ingredients, recent models are reasonably successful in reproducing the observed evolution of the rotational distributions of solar-type and lower mass stars on the main sequence (e.g. Irwin et al. 2008).

\section{Magnetic star-disk coupling during the pre-main sequence}

Since internal differential rotation plays a key role in the evolution of the surface rotation rate of solar-type stars, and because the amount of core-envelope decoupling in ZAMS stars is unknown, initial conditions for angular momentum evolution models have to be set at the very start of pre-main sequence (PMS) evolution, when the stars are still fully convective and may thus be assumed to be in solid-body rotation. The derivation of statistically robust rotational distributions for low-mass PMS stars at an age of $\sim 1 \mathrm{Myr}$, the so-called T Tauri stars (TTS), has been the goal of numerous studies over the past 20 years.

While the extrapolation of the Skumanich law back in time from the Sun's age to $1 \mathrm{Myr}$ predicted that TTS should have velocities as high as $150 \mathrm{~km} \mathrm{~s}^{-1}$, early measurements showed instead that they were relatively slow rotators, with $v_{\text {rot }} \cdot \sin i$ values of order of $20 \mathrm{~km} \mathrm{~s}^{-1}$ or less (Vogel \& Kuhi 1981; Bouvier et al. 1986; Hartmann et al. 1986). Hartmann \& Stauffer (1989) further pointed out that young stars accreting high angular momentum material from their circumstellar disk ought to spin up at velocities close to break-up on a timescale of order of 1 Myr. Obviously, some extremely efficient braking mechanism must be at work in these stars in order to prevent them from spinning up during their early PMS evolution.

Following a model originally proposed by Ghosh \& Lamb (1979) in the context of accreting neutron stars, Camenzind (1990) and Königl (1991) suggested that the magnetic coupling between the young active star and its inner accretion disk could lead to angular momentum transport that would effectively extract angular momentum from the central star, thus braking it. A key observational result in support to this hypothesis was that accreting TTS appeared to have, on average, lower rotation rates than non-accreting ones (Edwards et al. 1993; Bouvier et al. 1993). Somewhat paradoxically, it thus seemed that slow rotation was intimately linked to disk accretion in young stars.

Since then, evidence has been growing in support of magnetic star-disk coupling in young stars (Alencar 2007; Bouvier et al. 2007a, 2007b) and a number of theoretical and numerical studies have investigated how such a magnetic coupling could brake the young star much more efficiently than a solar-type magnetized wind would (e.g. Shu et al. 2007; Romanova et al. 2007; Matt \& Pudritz 2008). Additional evidence has been reported in the Spitzer era for slower rotation rates in accreting TTS (Rebull et al. 2006; Cieza \& Baliber 2007). As early envisionned by Choi \& Herbst (1996), the rotational period distribution of young stars appears bimodal, consisting of a peak of slow rotators at $P \sim 8$ days, and a wide tail 
of faster rotators. Slow rotators with infrared excess are assumed to be stars magnetically locked to their disk, and thus prevented from spinning up. Fast rotators without infrared excess have already dissipated their disk and have had enough time to spin up as they contract towards the main sequence.

Then again, albeit in quite a different way, magnetic fields, and more specifically the magnetic star-disk interaction, seem to dictate the angular momentum evolution of young stars during the PMS. Observationally, the lifetime of circumstellar disks is found to vary from star to star, over the range $11-10$ Myr (Hillenbrand 2005; Meyer et al. 2007). Stars with long-lived disks are prevented from spinning up for a significant fraction of their PMS evolution and consequently reach the ZAMS as slow rotators. On the opposite, stars with short-lived disks freely spin up as they contract towards the ZAMS, which they reach as fast rotators as solar-type magnetic winds are unable to brake them on such short timescales (cf. Matt \& Pudritz 2007). Thus, the wide dispersion of rotational velocities observed on the ZAMS for solar-type stars $\left(\leq 20-150 \mathrm{~km} \mathrm{~s}^{-1}\right.$, see Sect. 2) largely results from the distribution of disk lifetimes in the PMS (Bouvier et al. 1993; Bouvier 1994; Collier Cameron et al. 1995).

\section{Angular momentum evolution models}

Current angular momentum evolution models include the various physical mechanisms described in previous sections, albeit usually in a parametrized and/or phenomenological way. A grid of rotational models is shown for solar-mass stars in Fig. 2. The main phases of the evolution of surface rotation are clearly seen. As long as the star is coupled to its disk during the early PMS, it is assumed to evolve at a constant angular velocity. Once the disk has dissipated, a saturated braking law is applied, but solar-type winds are inefficient to prevent the star from spinning up as it contracts towards the ZAMS. Once on the ZAMS, the structure of the star stabilizes and magnetic braking becomes dominant. The assumed braking law leads to a convergence towards slow rotation within a few $100 \mathrm{Myr}$ on the main sequence. Indeed, the surface rotation rate of mature solar-type stars has lost memory of the past rotational history.

These models are confronted to observations in Figure 3. In the last years, rotational periods have been measured for hundreds of low-mass stars in molecular clouds (1-5 Myr) and young open clusters (40-600 Myr), thus providing a tight observational sampling of the rotational evolution of low-mass stars (e.g. Irwin et al. 2008). Figure 3 shows these rotational period distributions, converted to angular velocity $(\omega=2 \pi / P)$, for solar-type stars in a number of young and intermediateage clusters (see Bouvier 2008 for complete references on the datasets). The 2 models shown in Fig. 3illustrate the evolution of the slowest and fastest rotators. For the sake of clarity, models for intermediate rotators are not shown, but would have a similar behaviour, with parameters intermediate between those of slow and fast rotator models.

The model for fast rotators starts from an initial period of $1.2 \mathrm{~d}$. The star is assumed to remain coupled to its disk for $5 \mathrm{Myr}$, then spins up to a velocity of 


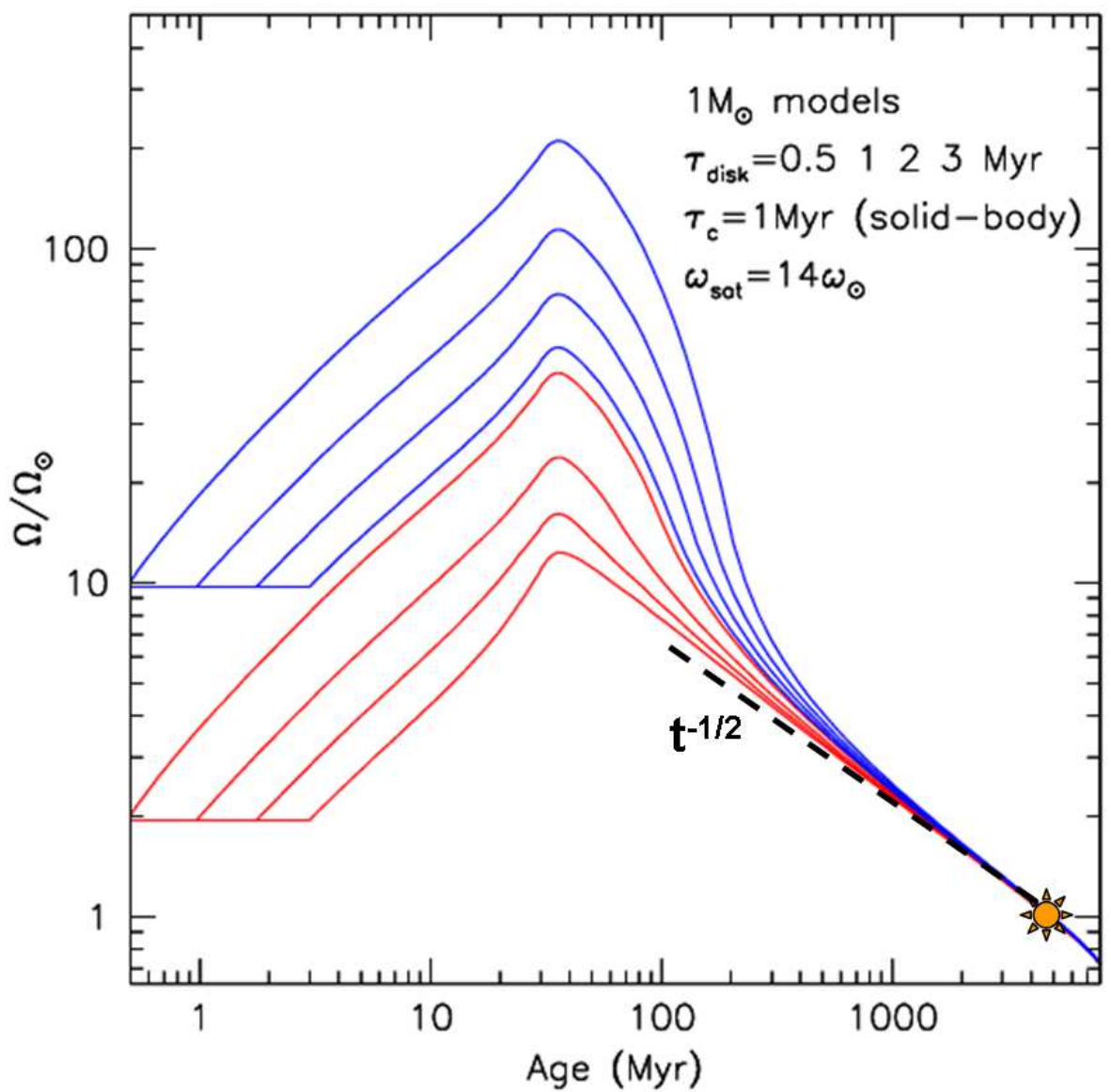

Fig. 2. Grid of rotational models for $1 \mathrm{M}_{\odot}$ stars. Starting from 2 initial velocities, rotational tracks are computed for disk lifetimes of $0.5,1,2$, and $3 \mathrm{Myr}$ (from left to right). These models assume a core-envelope coupling timescale of $1 \mathrm{Myr}$, which is equivalent to solid-body rotation. The velocity at which dynamo saturation occurs is taken to be $14 \Omega \odot$. Note the convergence towards uniformly slow rotation at the Sun's age, regardless of the past rotational history. The $\mathrm{t}^{-1 / 2}$ Skumanich relationship is shown for reference. The $1 \mathrm{M}_{\odot}$ structural evolution model is from Baraffe et al. (1998).

order of $160 \mathrm{~km} \mathrm{~s}^{-1}$ on the ZAMS, and is eventually spun down by a magnetized wind on the MS to the Sun's velocity. This model fits reasonably well the PMS spin up and the rapid MS spin down observed for fast rotators between 5 and $500 \mathrm{Myr}$. In order to reach such an agreement, the core-envelope coupling timescale has to be short, $\tau_{c} \sim 10 \mathrm{Myr}$. A longer coupling timescale would lead to envelope spin 

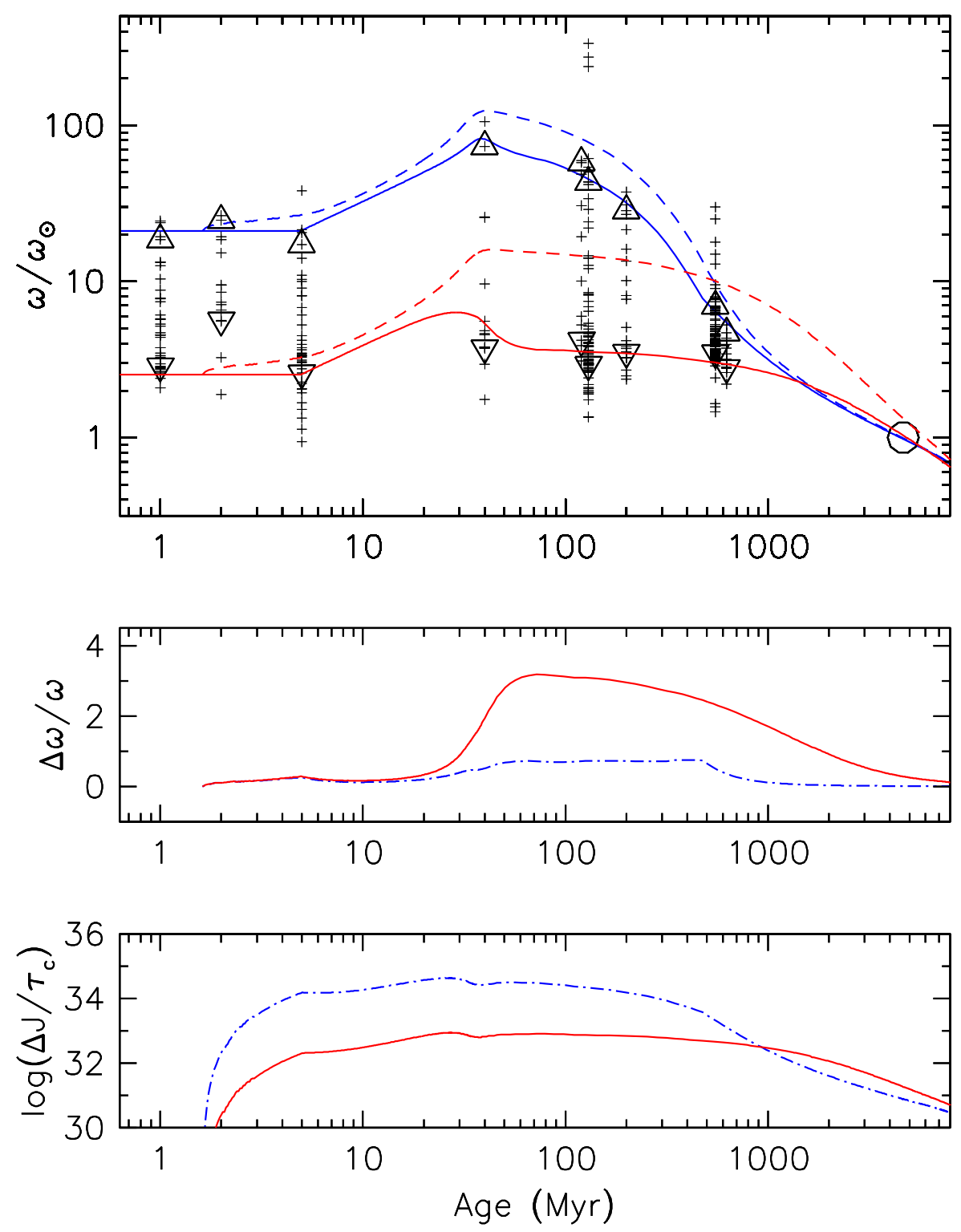

Fig. 3. Rotational models for slow and fast solar-mass rotators. Upper panel : observations and models. The 10th and 75th percentiles of the observed rotational period distributions of solar-type stars $\left(0.8-1.1 \mathrm{M}_{\odot}\right)$ were converted to angular velocity and are plotted as direct and inverted triangles as a function of time. Individual measurements of rotational periods converted to angular velocities are also shown in order to illustrate the statistical significance of the various samples. The modeled evolution of surface rotation for slow and fast rotators is shown by the solid lines. For both models, the rotation of the radiative core is shown by the dashed lines. With a core-envelope coupling timescale of only $10 \mathrm{Myr}$, little differential rotation develops in fast rotators. In contrast, the 100 Myr core-envelope coupling timescale in slow rotators leads to the development of a large velocity gradient at the base of the convective zone. A disk lifetime of $5 \mathrm{Myr}$ is assumed for both models. Lower panels : The velocity shear at the base of the convective zone, $\left(\omega_{\text {rad }}-\omega_{\text {conv }}\right) / \omega_{\text {conv }}$, and the angular momentum transport rate, $\Delta J / \tau_{c}\left(\mathrm{gcm}^{2} s^{-2}\right)$, from the core to the envelope are shown for slow (solid line) and fast (dotted-dashed line) rotators. From Bouvier (2008). 
down before the star reaches the ZAMS, and a slower spin down rate on the early MS, both of which would conflict with observations. The tight coupling between the core and the envelope implies that little differential rotation develops in fast rotators, with the rotation of the core barely exceeding that of the envelope on the early MS (see Fig. 3).

The slow rotator model has an initial period of $10 \mathrm{~d}$ and the star-disk magnetic interaction is assumed to last for $5 \mathrm{Myr}$ in the early PMS. As the star approaches the ZAMS, both the outer convective envelope and the inner radiative core spin up. Once on the ZAMS, however, only the outer envelope is quickly braked, while the core remains in rapid rotation. This behaviour results from an assumed weak coupling between the core and the envelope, with $\tau_{c} \sim 100 \mathrm{Myr}$. On the early MS, the rapidly-rotating core transfers angular momentum back to the envelope, which explains the nearly constant surface velocity over several 100 Myr in spite of magnetic braking. A long coupling timescale between the core and the envelope is thus required to account for the observed rotational evolution of slow rotators. A long $\tau_{c}$ in slow rotators implies inefficient transport of internal angular momentum and results in a large velocity gradient at the core-envelope boundary (see Fig. (3).

The main difference between fast and slow rotators in these models is thus twofold : the initial angular momentum and the level of core-envelope decoupling. The initial angular momentum of fast rotators is 10 times higher than that of slow ones. Fast rotators lose more angular momentum over the course of their evolution than slow rotators, as they both converge towards the Sun's velocity at 4.65 Gyr. The lower panels in Fig. 3 show the magnitude of the rotational shear at the base of the convective envelope for slow and fast rotators, and the amount of angular momentum transported from the core to the envelope. Strong differential rotation develops in slow rotators at the ZAMS and remains large during early MS evolution until the core and the envelope eventually recouple after a few Gyr. Clearly, slow rotators exhibit a much larger rotational shear at the base of their convective envelope than fast rotators during most of their evolution. Conversely, angular momentum transport from the core to the envelope is much more efficient in fast rotators than in slow ones, which results in little differential rotation indeed. Rotational shear and angular momentum transport are both directly related to the assumed core-envelope coupling timescale and are independent of the disk lifetime. The implications of the different internal rotation profiles between slow and fast rotators for the properties of mature solar-type stars and for the planet formation process in circumstellar disks have been discussed in Bouvier (2008).

\section{Conclusions}

Stellar magnetic fields are the central ingredient governing the rotational evolution of solar-type stars. Star-disk magnetic coupling during pre-main sequence evolution dictates the rotational history of young suns, up to their arrival on the zero-age main sequence. Magnetized stellar winds then become dominant on the main sequence and yield uniformly slow rotation by the age of the Sun. Semiempirical models are relatively successful in reproducing the main trends of the 
observed rotational evolution of solar-type stars from their birth to the Sun's age. Yet, these models merely include phenomenological descriptions of the physical processes at work. An area of progress for the years to come will hopefully be the development of complete physical theories for the star-disk magnetic interaction, for angular momentum loss through magnetized winds, and for instabilities that redistribute angular momentum in stellar interiors. On the observational side, spectropolarimetric measurements will provide unprecedented details on the strength and topology of stellar magnetic fields in solar-type stars at various ages (see Donati, this volume). Such observations will shed light on the nature of the star-disk interaction in young stars (Donati et al. 2008), provide physical clues to the empirical concept of dynamo saturation (Petit et al. 2008), and help extrapolating the angular momentum evolution models developed for solar-type stars to lower mass stars (Morin et al. 2008).

\section{References}

Alencar, S. H. P. 2007, IAU Symposium, 243, 71

Allain, S. 1998, A\&A, 333, 629

Baraffe, I., Chabrier, G., Allard, F., \& Hauschildt, P. H. 1998, A\&A, 337, 403

Belcher, J. W., \& MacGregor, K. B. 1976, ApJ, 210, 498

Bouvier, J. 1994, Cool Stars, Stellar Systems, and the Sun, 64, 151

Bouvier, J. 1997, Memorie della Societa Astronomica Italiana, 68, 881

Bouvier, J. 2008, A\&A, 489, L53

Bouvier, J., et al. 2007a, A\&A, 463, 1017

Bouvier, J., Forestini, M., \& Allain, S. 1997, A\&A, 326, 1023

Bouvier, J., Bertout, C., Benz, W., \& Mayor, M. 1986, A\&A, 165, 110

Bouvier, J., Alencar, S. H. P., Harries, T. J., Johns-Krull, C. M., \& Romanova, M. M. 2007b, Protostars and Planets V, 479

Bouvier, J., Cabrit, S., Fernandez, M., Martin, E. L., \& Matthews, J. M. 1993, A\&A, 272,176

Camenzind, M. 1990, Reviews in Modern Astronomy, 3, 234

Choi, P. I., \& Herbst, W. 1996, AJ, 111, 283

Cieza, L., \& Baliber, N. 2007, ApJ, 671, 605

Collier Cameron, A., \& Jianke, L. 1994, MNRAS, 269, 1099

Collier Cameron, A., Campbell, C. G., \& Quaintrell, H. 1995, A\&A, 298, 133

Donati, J.-F., et al. 2008, MNRAS, 386, 1234

Durney, B. R., \& Latour, J. 1978, Geophysical and Astrophysical Fluid Dynamics, 9, 241

Edwards, S., et al. 1993, AJ, 106, 372

Endal, A. S., \& Sofia, S. 1981, ApJ, 243, 625

Ghosh, P., \& Lamb, F. K. 1979, ApJ, 234, 296

Hartmann, L., \& Stauffer, J. R. 1989, AJ, 97, 873

Hartmann, L., Hewett, R., Stahler, S., \& Mathieu, R. D. 1986, ApJ, 309, 275

Hillenbrand, L. A. 2005, arXiv:astro-ph/0511083 
Irwin, J., Hodgkin, S., Aigrain, S., Bouvier, J., Hebb, L., \& Moraux, E. 2008, MNRAS, 383,1588

Kawaler, S. D. 1988, ApJ, 333, 236

Keppens, R., MacGregor, K. B., \& Charbonneau, P. 1995, A\&A, 294, 469

Königl, A. 1991, ApJl, 370, L39

Kraft, R. P. 1970, Spectroscopic Astrophysics. An Assessment of the Contributions of Otto Struve, 385

Krishnamurthi, A., Pinsonneault, M. H., Barnes, S., \& Sofia, S. 1997, ApJ, 480, 303

MacGregor, K. B., \& Brenner, M. 1991, ApJ, 376, 204

Matt, S., \& Pudritz, R. E. 2008, ApJ, 681, 391

Matt, S., \& Pudritz, R. E. 2007, IAU Symposium, 243, 299

Meyer, M. R., Backman, D. E., Weinberger, A. J., \& Wyatt, M. C. 2007, Protostars and Planets V, 573

Morin, J., et al. 2008, MNRAS, 390, 567

Petit, P., et al. 2008, MNRAS, 388, 80

Pinsonneault, M. H., Kawaler, S. D., Sofia, S., \& Demarque, P. 1989, ApJ, 338, 424

Rebull, L. M., Stauffer, J. R., Megeath, S. T., Hora, J. L., \& Hartmann, L. 2006, ApJ, 646, 297

Romanova, M. M., Long, M., Kulkarni, A. K., Kurosawa, R., Ustyugova, G. V., Koldoba, A. K., \& Lovelace, R. V. E. 2007, IAU Symposium, 243, 277

Schatzman, E. 1962, Annales d'Astrophysique, 25, 18

Schatzman, E. 1959, The Hertzsprung-Russell Diagram, 10, 129

Shu, F. H., Galli, D., Lizano, S., \& Cai, M. J. 2007, IAU Symposium, 243, 249

Skumanich, A. 1972, ApJ, 171, 565

Soderblom, D. R. 1983, ApJs, 53, 1

Stauffer, J. R., Hartmann, L. W., Burnham, J. N., \& Jones, B. F. 1985, ApJ, 289, 247

Stauffer, J. R., Caillault, J.-P., Gagne, M., Prosser, C. F., \& Hartmann, L. W. 1994, ApJs, 91, 625

Vilhu, O. 1984, A\&A, 133, 117

Vogel, S. N., \& Kuhi, L. V. 1981, ApJ, 245, 960

Weber, E. J., \& Davis, L. J. 1967, ApJ, 148, 217 